\title{
EDITORIAL
}

\section{Lessons from Crew Resource Management for Cardiac Surgeons}

\author{
Patrick Marvil, BS, Curt Tribble, MD
}

Division of Thoracic and Cardiovascular Surgery, University of Virginia Health System, Charlottesville, Virginia, USA

\section{INTRODUCTION}

Crew resource management (CRM) describes a system developed in the late 1970s in response to a series of deadly commercial aviation crashes. This system has been universally adopted in commercial and military aviation and is now an integral part of aviation culture. CRM is an error mitigation strategy developed to reduce human error in situations in which teams operate in complex, high-stakes environments. Over time, the principles of this system have been applied and utilized in other environments, particularly in medical areas dealing with high-stakes outcomes requiring optimal teamwork and communication. While the data from formal studies on the effectiveness of formal CRM training in medical environments have reported mixed results, it seems clear that some of these principles should have value in the practice of cardiovascular surgery.

From a broader perspective, why might the principles of CRM be useful in health care? There is teamwork in almost every setting in healthcare, and CRM is an error reduction strategy designed to optimize the performance of teams in high-stakes situations. The Institute of Medicine, in its report "To Err Is Human," estimated that 44,000 to 98,000 deaths per year in the US are caused by medical errors. As a system and as individuals, medical practitioners must strive to reduce the morbidity and mortality of errors by reducing their incidence.

The authors, a former military aviator who is now a medical student (PCM) and a cardiovascular surgeon (CGT) with nearly 40 years of experience in medicine and surgery, have recently compared their experiences in these realms and herein suggest some strategies developed during the evolution of CRM that are likely to be useful and effective in the practice of cardiovascular surgery. We will present the principles of CRM as learned and utilized in recent military deployments by one author (PCM) and suggest ways that these principles are already being or might be applied to modern cardiovascular surgical teams (CGT).

While the principles of CRM are often laid out as seven principles, we will outline them as four tenets, and then

\footnotetext{
Correspondence: Curt Tribble, MD, Professor of Cardiothoracic Surgery, University of Virginia Health System, Division of Thoracic and Cardiovascular Surgery, Box 800679, Charlottesville, VA, 22908; 434-243-9250 (e-mail: CGT2E@bscmail.mcc.virginia.edu).
}

describe approaches to put these principles to use in cardiovascular surgery. These tenets include:

- Mission analysis

- Leadership

- $\quad$ Communication \& assertiveness

- Situational awareness, decision making \& adaptability

A review of the available literature on the application of principles of CRM in medical environments is summarized as follows.

In the early 2000s, there were only a few studies exploring the use of CRM in healthcare. Within the last several years, an increasing number of pertinent studies have been published. In "Briefing and Debriefing in the Operating Room Using Fighter Pilot Crew Resource Management," McGreevy and Otten make comparisons between the personalities of fighter pilots and surgeons, explain the form and content of a fighter pilot brief and debrief, and make recommendations for applying the fighter pilot brief and debrief to the operating room [McGreevy 2007]. Their suggestions for briefing in the operating room include: giving background on a patient to identify each as an individual and not just a routine, generic case; to provide an overview of the impending operation tailored to complexity (short for a routine case, longer and more in depth for a complex case); and to lay out the "mission objectives" with some detail of how the operation will proceed and what could go wrong at various points, with the intent of engaging participants according to their level of experience. They also suggest that a specific learning objective for each case could be shared. Their recommendations for debriefing after the operation include: a timely, short discussion of what went well and what did not, with the goal of praising good performance and avoiding repeat poor performance. The authors suggest that videotapes of the operations could be used for structured review to help improve future performance. The authors also cite the need for a cultural change among surgeons to embrace mistakes and to strive for improvement through self-reflection.

In a study titled "Crew Resource Management: Using Aviation Techniques to Improve Operating Room Safety," mandatory CRM training was implemented for surgeons, nurses, anesthesiologists, and operating room technicians and assistants at an academic medical center [Grogan 2004]. CRM techniques such as pre-op checklists and briefings, post-op debriefings, and read-and-initial files were implemented. A 
read-and-initial file is a way to disseminate information to ensure that the teams are up to date on chosen topics. The results presented in this paper showed that wrong-site surgeries and retained foreign bodies decreased from seven to zero over one year. Malpractice expenses decreased from $\$ 793,000$ over four years to zero dollars over the next four years. Sustainment training in CRM was not part of the plan, and briefs and debriefs waned as a result. Wrong site surgeries and retained foreign bodies increased as the time interval after the original CRM training lengthened.

A study titled "Aviation and Healthcare: a Comparative Review with Implications for Patient Safety" that analyzed the convergence and divergence in the realms of aviation and healthcare concluded that the transfer of lessons from aviation to healthcare has to be nuanced to optimize the usefulness of the principles of CRM in healthcare. [Kapur 2015]. Another conclusion of this paper was that healthcare should emulate aviation by involving specialists in human factors and in the psychological aspects of patient safety.

A consistent theme of these papers is that CRM might gain additional traction in healthcare when it can be shown to affect the bottom line. A study that addressed that issue entitled "What is the Return on Investment for Implementation of a Crew Resource Management Program at an Academic Medical Center?" describes a health-system-wide implementation of CRM with a focus on the return on investment. The cost of the training was $\$ 3.6$ million over three years. The return on investment was defined as a reduction in avoidable events with an estimate of the potential for cost savings. Savings to the medical center were estimated to be between $\$ 12.6$ and \$24.4 million over three years. The authors of this study concluded that the return on investment for implementing CRM could be quite significant [Moffatt-Bruce 2017].

\section{MISSION ANALYSIS}

Mission analysis is defined as the process of developing shortterm, long-term, and contingency plans while coordinating, allocating, and monitoring resources. In military aviation, there are frameworks for analyzing commonly executed missions. An example of such a mission is the ship to shore helicopter insertion of troops into a combat zone. Military pilots start with these frameworks and fill in details for the particular mission at hand based on friendly and enemy situations on the battlefield, weather, distances to be flown, nighttime illumination, etc. Thorough preparation removes as much uncertainty as possible for the conduct of the mission and includes planning as detailed as where a pilot would expect to see the moon on the horizon when making a turn at a route checkpoint. Military pilots organize and plan not only for what will occur but also for what may occur to remove as much uncertainty as possible. Decisions regarding "go/no-go" criteria are made in the ready room before a mission begins to ease the decision-making burden of the mission commander in flight, and to prevent a bias towards continuing a mission in the heat of the moment if circumstances change and dictate that the mission should be aborted.

During mission execution, resources available are continuously monitored and coordinated to optimize their contribution towards the mission. If resources drop below a certain threshold and mission accomplishment becomes out of reach, then the decision is made to abort the mission. For example, troop transport helicopters need armed escorts from either attack helicopters or fighter jets. If armed escort becomes inadequate, then the exposure risk to the transport helicopters might be judged too high to complete the mission.

The principles of mission analysis as applied in cardiovascular surgery are readily apparent in the growing use of multidisciplinary teams to make decisions about programs as well as about individual patients. While some of the first of these multidisciplinary teams were developed in the transplant arena, they are now becoming common in other areas of thoracic and cardiovascular medicine and surgery, such as tumor boards, valve teams, and heart teams focused on ischemic cardiac disease. It is our observation that these types of teams are very valuable but are likely under-utilized currently. A strategy that can be employed in this team environment is called scenario planning, which has the objective of outlining as many of the possible outcomes and issues as possible, with the goal of being as prepared as is feasible for these issues.

At a more granular level, planning for individual cardiovascular surgical cases that involve large, multidisciplinary teams requires the sharing of detailed information. It has been estimated that about 100 different individuals play an important role in the care of each cardiac surgical patient. When all of these individuals are notified of the plan, they can each communicate efficiently with the rest of the protagonists to ask questions or offer suggestions. We have implemented this aspect of CRM by using email to circulate plans the night before a planned procedure. We have found this strategy to be very useful [Tribble 2016a].

The next use of the CRM principle of mission analysis in cardiovascular surgery is the timeout that now routinely occurs in the operating room, just prior to the start of the planned operation. We have found it valuable to not only review the plans and possible issues that might arise, but to also ensure that any supplies or equipment will be available when needed, especially if these items are somewhat unusual. A particularly useful and effective part of the timeout in the operating room is to identify each member of the operating room team by their first names. Furthermore, it is worth stating during the timeout that even the most inexperienced participants may see or think of something that others are overlooking, and they are encouraged to speak up if that occurs. A particularly empowering way to describe this possibility is to say explicitly that everyone in the room will obviously have a slightly different view of what is going on in the case and, therefore, each participant should be encouraged to "say something if you see something" [Calland 2002].

The principle of debriefing in CRM can be employed in both an informal and formal manner. The informal process of reflection and debriefing can be accomplished both by initial verbal brainstorming and feedback right after the operation and by encouraging participants, especially the primary protagonists (such as the primary surgeon and the assistants), to write out their reflections in notebooks. An example of a more formal process of debriefing is the Morbidity and Mortality 
conferences that are almost universally used in surgical services. As Atul Gawande once wrote, "it isn't reasonable to ask that we achieve perfection. What is reasonable to ask is that we never cease to aim for it." [Tribble 2016b].

\section{LEADERSHIP}

Leadership in CRM includes directing and coordinating personnel, stimulating teamwork, delegating tasks, laying expectations, focusing resources on crucial aspects of the mission, providing feedback, and creating and maintaining a professional atmosphere.

In the military, a commander lays out his or her commander's intent, which is the desired end result for a given mission. This process allows everyone involved to focus and allocate resources based on a granular assessment of the primary goal of the mission. Once the commander's intent is laid out, in theory the mission can be accomplished without further input from the commander, as might be the case if the commander becomes a casualty or the lines of communication are disrupted. While the commander's focus is generally on big-picture expectations, more detailed expectations can also be set in order to optimize the chances for success.

A leader needs to provide feedback on how his or her expectations are being met as the mission progresses, to allow those in the chain of command the opportunity to adjust their efforts. For example, this feedback could take the form of a one-phrase radio transmission regarding the shape of a formation of helicopters. A leader should strive to set the tone for the whole mission, which will create or erode the professional atmosphere depending on the approach utilized. Proper planning, preparation, and competence regarding the mission at hand are all parts of setting the tone for a professional organization.

It has been recognized that there are two types of leadership in an aircrew. Designated leadership is leadership by authority, position, rank, or title. Functional leadership is leadership by knowledge or expertise, and it allows the most qualified individual to take charge of a situation temporarily. For example, an enlisted crew chief in the back of a helicopter might have many more years of experience than either pilot in the cockpit, and his expertise needs to be valued and incorporated in the trajectory of the mission.

Caring for patients who require cardiovascular surgical operations is clearly a team activity. Everyone on these teams plays a vital role in achieving optimal outcomes for the patients. As noted earlier, these teams include a surprisingly large number of practitioners. As the leader of these large, complex, and highly professional teams, the cardiovascular surgeon must treat all members of these teams with the respect that they are due. It is also essential to convey a sense of optimism that a procedure has a chance-probably even a good chance in most circumstances - of helping the patient achieve a good outcome, particularly if everyone involved does their part conscientiously and well. This sense of optimism can be infectious. We often remind our team members of the old saying that "the vast majority of our patients will do well, though not by much," which conveys the appropriate optimism while reminding all involved that everything matters and nothing is neutral. On a similar note, the leaders of such teams should always strive to use the language of commitment, while avoiding the language of complaint [Tribble 2014].

\section{COMMUNICATION AND ASSERTIVENESS}

Communication is at the heart of any team effort, as good communication is essential for a team to be effective. However, it takes training, practice, and thought to be an effective communicator. Communication involves clearly and accurately sending and acknowledging information, instructions, commands, and feedback. As a sender one must be accurate and timely. Pilots think about what they are going to say over the radio before they say it, to minimize transmission time while clearly communicating critical information. There can be no room for misinterpretation, and nothing can be left to the imagination.

As a receiver one must acknowledge and perhaps repeat, rephrase, clarify, or provide feedback. This is the second part of the two-way process. A pilot instructs his or her copilot and expects a response acknowledging, understanding, and with intent to comply. For example, if a pilot instructs the copilot to descend from 100 feet to 50 feet during a low-level flight, the copilot might acknowledge with something as simple as, "Roger, down to 50 feet." Conversely, the pilot must also acknowledge communication from the copilot to endorse the validity of the copilot's input to the accomplishment of the mission. Another aspect of communication is timeliness. A military pilot who was shot at from a certain location on the ground needs to inform the air control agency and other aircraft operating within the same area as soon as possible. If the pilot waits too long after the incident, then other aircraft may be exposed to the danger of flying over the threat area.

Assertiveness, as defined in CRM, is providing information without being asked, making suggestions, asking questions, maintaining one's position until facts prove that a different course is needed, and stating opinions in a respectful manner. In aviation, there can be a cockpit gradient. When there is a senior pilot and a junior copilot, the junior copilot might be reluctant to speak up and state an opinion or state that he or she senses that something is wrong. The senior pilot might have treated the more junior pilot in such a way that the more junior pilot is reluctant to speak up. Before the principles of CRM became commonly used, there was often a steep power gradient in the cockpit, in which the senior pilot would not be questioned. Rank and experience both can contribute to this cockpit gradient. CRM dictates that it is up to the senior pilot to level this gradient both when laying out the expectations prior to flight as well as through actions taken during the flight. Junior pilots are taught that they have the responsibility of voicing their opinions and providing input in a respectful, timely manner.

Another phenomenon that can interfere with assertiveness in aviation is called the sandbag syndrome. This entity is described as the feeling of comfort that can arise when someone else seems to have a situation under control. A junior pilot can easily drift into trusting the senior pilot in a scenario such as a difficult instrument approach under conditions of low visibility and ceilings. The copilot might think that a deviation from course or altitude is intentional, when in fact the senior pilot might be suffering 
from vertigo and should, therefore, be verbally challenged if deviations from normal protocol become apparent. A complementary approach might be the "trust but verify" approach, in which a junior pilot inherently trusts the experience of someone more senior but remains ready to speak up when something is happening that does not seem appropriate.

One of the important principles of optimal communication in a cardiovascular operating room is to adhere to the CRM strategy of call and response. For instance, it has been said that those actually doing these operations are living life in a coffee can, by which we mean that one must maintain one's visual focus on a very small operative field. However, the surgeon must communicate effectively and reliably with the other five or so groups who are also working with them. These groups include the anesthesiologists, the perfusionists, the scrub nurses, the circulating nurses, and the physicians' assistants. One of the CRM principles commonly used to optimize communication during these complex cases is that of call and response. For instance, if the surgeon asks that the flow of the heart lung machine be reduced, the perfusionist is expected to respond by repeating the request to acknowledge its accurate reception. Another CRM principle that should be adhered to in the cardiovascular operating room is the reduction in hierarchy. This leveling of the environment can be accomplished in many ways including the use of less authoritarian or hierarchal language, such as by using first names, and by encouraging the active participation of all in the room in offering observations or suggestions. When addressing differences of opinion, one must constantly employ the venerable tradition of respectful argumentation. A valuable way to think constructively about differing views is to ask oneself what might be true about what someone has suggested [Tribble 2014].

\section{SITUATIONAL AWARENESS, DECISION MAKING, ADAPTABILITY}

In CRM, situational awareness is described as the degree of accuracy by which perception mirrors reality. Situational awareness also includes detecting and commenting on deviations and identifying and acknowledging problems. One way to prepare for having good situational awareness is by a thorough briefing prior to the start of the mission. Thorough does not mean that it has to take more than a few minutes. Being thorough involves talking through the critical things that will be needed, the critical points in a mission, and verifying the contingencies and expectations in order to increase each team member's situational awareness. In order to maintain situational awareness one must continuously use all available information to update and revise the perceptions of the team members.

Another principle of CRM is to avoid a fixation on one piece of data while ignoring other available data points. An example of this pitfall occurred during the departure phase of a routine maintenance flight (to set up and test aircraft systems) in Iraq, when one of the authors (PCM), his copilot, and even the crew chief in the back of the helicopter became engrossed in a fluctuating engine gauge. All three were staring at and discussing the engine performance, and no one was focused on flying the aircraft. The weather was marginal with heavy mist reducing visibility and obscuring the horizon, and the departure path took the helicopter over a large lake very soon after takeoff. While the three crew members were focusing on the engine gauge, the aircraft was descending towards the water. The pilot and copilot did not notice both audible and visual warnings regarding the helicopter's low and continuously decreasing altitude, and the weather reduced the normal visual cues for this descent that might have been picked up in the pilots' peripheral vision. Eventually the copilot glanced at the radar altimeter and, realizing the very low altitude, pulled power to the engines to arrest the descent and begin climbing, a scant few seconds before a well-functioning helicopter would have impacted the water.

It has been asserted that cardiovascular surgeons make a life or death decision approximately every 10 seconds during a typical cardiovascular operation. In fact, most would agree that about $75 \%$ of great surgical outcomes are attributable to good judgment while only $25 \%$ are dependent on technique. Obviously, all on the team must maintain an awareness of all that is going on in the room at all times, including the activities of the anesthesia team, the perfusionists, the physicians' assistants, and the nursing team. However, at times the surgeons must have intense focus on the technical task at hand, potentially blocking out most, if not all, stimuli in the room. Thus, one of the skills the surgeons must develop in the realm of situational awareness is the ability to cycle regularly from the immediate task of the moment back to a more global awareness of the conditions and activities of the room. That is, one must be able to develop a rhythm of shifting between focus and awareness. Another instinct we try to inculcate in our cardiac surgical trainees is the need to think constantly on what one will do next, particularly if the original plan does not seem to be unfolding as planned.

Another aspect of the situational awareness principle in CRM is to avoid distractions. One of the most subtle types of distractions in a cardiovascular operating room is an uncommon condition. An example of this type of distraction has been described by astronauts as becoming "space stupid" when working under conditions of weightlessness, where objects are no longer affected by gravity. While ordinary cardiovascular operations will not include a condition as unusual as weightlessness, there are certainly times when an unusual or unexpected circumstance arises that can throw the team out of its usual rhythm. In such situations, one must recognize and, if possible, regain control of the distraction to avoid allowing it to negatively affect the tasks of the moment. When such distractions do inevitably arise, one does have a choice of how one will react, and it is optimal to strive to maintain grace under pressure while regaining control of the situation.

\section{Summary}

In the end, pilots are not doctors and nurses are not flight crew, but there seem to be more similarities than differences. In looking at a few characteristics of pilots and their standardized approach to missions, we can draw some parallels between them and cardiovascular surgeons. Both groups may have unrealistic attitudes regarding the effect of stressors on 
their performance, such as the belief that their personal problems do not affect their work. This sense of personal invulnerability is a negative aspect of the culture and can lead to degraded performance.

As we come to accept that errors are an inevitable result of natural human limitations of performance in complex systems, we can look for mitigation strategies for those errors. It can be difficult in healthcare to admit errors when those errors directly impact patients' outcomes and lives. However, we must embrace the reality that error is inherent in our work in order to seek out means for its reduction. And the principles of CRM can be one of those means.

Further studies are required to better correlate CRM to outcomes that matter to the hospital administrators and healthcare managers, physicians, and nurses, which include both patient-oriented outcomes and financial considerations. The culture of medicine is becoming more open to errorreduction measures such as CRM in its attempts to progress towards providing the safest and most effective care possible. If the principles of CRM are to become more fully incorporated in healthcare, exposure to these principles should be introduced in any healthcare curriculum and continue throughout the careers of healthcare providers. CRM in aviation is in its sixth generation. What might the sixth generation of CRM in healthcare look like, and how much error reduction is possible by the time that sixth generation is reached?

As the principles of Crew Resource Management continue to be incorporated into medicine, in particular in surgical practices, cardiovascular surgeons and their teams can increasingly utilize these principles on a day-to-day basis to enhance the smooth functioning of their teams, even without extensive training and the substantial attendant institutional costs, though certainly some additional training within these complex teams would be useful. In this brief review, we have suggested ways that cardiovascular surgeons can apply many of the principles of Crew Resource Management to their practices for the benefit of their patients.

\section{REFERENCES}

Calland JF, Guerlain S, Adams RB, Tribble CG, Foley E, Chekan EG. 2002. A systems approach to surgical safety. Surg Endosc 16:1005-14.

Grogan E, Stiles R, France D, et al. 2004. The impact of aviation-based teamwork training on the attitudes of health-care professionals. J Am Coll Surg 199:843-8.

Kapur N, Parand A, Soukup T, Reader T, Sevdalis N. 2015. Aviation and healthcare: a comparative review with implications for patient safety. JRSM Open 7:2054270415616548.

McGreevy J, Otten T. 2007. Briefing and debriefing in the operating room using fighter pilot crew resource management. J Am Coll Surg 205:169-76.

Moffatt-Bruce S, Hefner J, Mekhjian H, et al. 2017. What is the return on investment for implementation of a crew resource management program at an academic medical center? Am J Med Qual 32:5-11.

Tribble C, Merrill W. 2014. The way we talk is the way we teach. J Thorac Cardiovasc Surg 147:1155-9.

Tribble C. 2016. Failing to prepare is preparing to fail. Heart Surg Forum 19:112-5.

Tribble C. 2016. A practical minded obsession with the possibility and consequence of failure. Heart Surg Forum 19:1-4. 